\title{
Comparison of respiratory navigator techniques for interleaved high-resolution coronary vessel wall imaging
}

\author{
Mehmet Akcakaya ${ }^{1 *}$, Markus Henningsson², Reza Nezafat ${ }^{1}$, Rene M Botnar ${ }^{2}$ \\ From 16th Annual SCMR Scientific Sessions \\ San Francisco, CA, USA. 31 January - 3 February 2013
}

\section{Background}

Three-dimensional high-resolution PSIR LGE [1], flowindependent vessel wall imaging [2], or T1 mapping [3] are all implemented with interleaved sequences, where the same volume is imaged multiple times with different inherent contrast. These volumes are then processed together to generate additional information, e.g. recovering the sign of magnetization, depicting vessel walls via subtraction, or calculating T1 maps via curve-fitting. Such techniques rely on correct registration of the voxels, hence on accurate motion compensation. In this study, we sought to explore the effects of synchronous and asynchronous navigator gating and tracking on respiratory motion compensation in high-resolution coronary vessel wall imaging with an interleaved T2prep sequence (i-T2prep).

\section{Methods}

The right coronary artery (RCA) of 7 subjects $(29.6 \pm 12.6$ yrs) were imaged on a $1.5 \mathrm{~T}$ Philips Achieva magnet with a targeted i-T2prep sequence using an interleaved SSFP acquisition $\left(\mathrm{TR} / \mathrm{TE} / \alpha=4.3 / 2.1 \mathrm{~ms} / 90^{\circ}\right.$, resolution $=1 \times 1 \times 3$ $\mathrm{mm}^{3}, \mathrm{FOV}=270 \times 270 \times 30 \mathrm{~mm}^{3}$ ) where one heartbeat (interleaf 1) was acquired with T2prep prepulse, and the subsequent one (interleaf 2) without [2]. A $5 \mathrm{~mm}$ gating window was used with the following strategies: 1) Accept the data only if both interleaves are within the gating window (synchronous), 2) Accept each interleaf independently (asynchronous). Acquisition time for each scan was recorded. Coronary vessel wall images were generated by weighted subtraction of the two interleaves [2], and quantitative vessel sharpness measurements were performed on these vessel wall images.

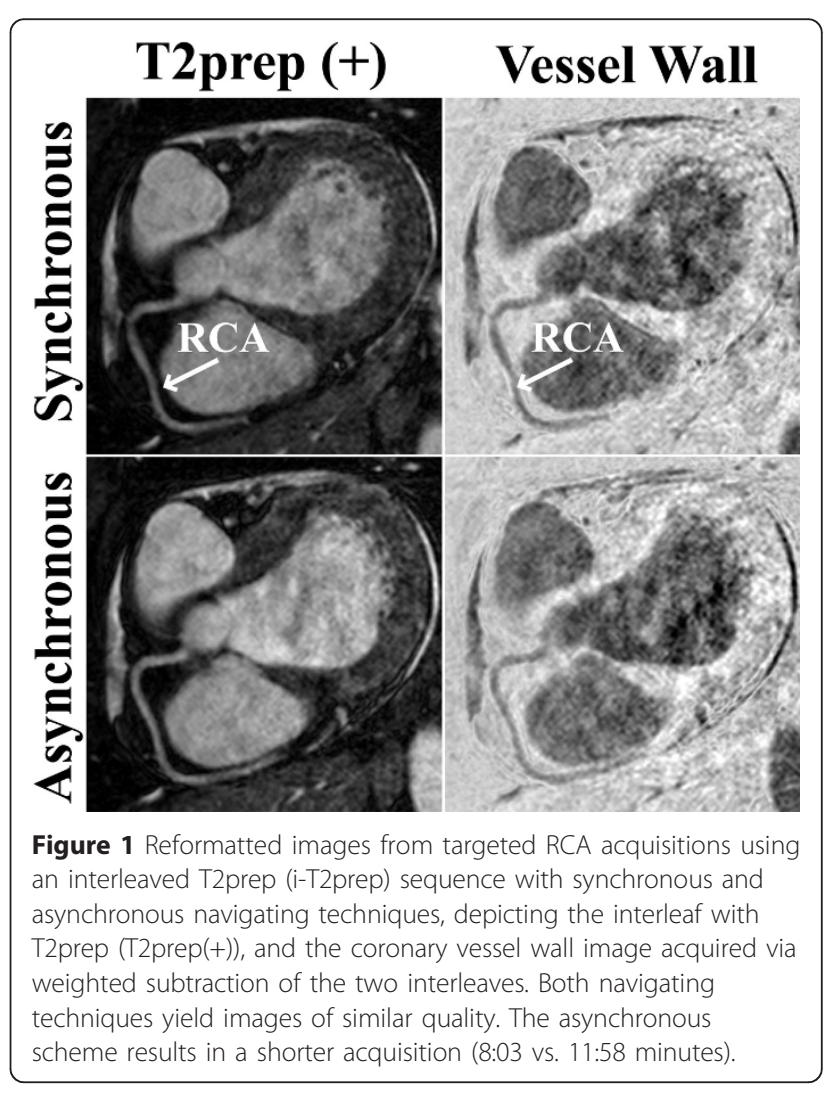

\section{Results}

Figure 1 shows reformatted RCA images with T2prep $(+)$ and vessel wall images from a healthy subject. Both navigating techniques provide similar image quality, but the 


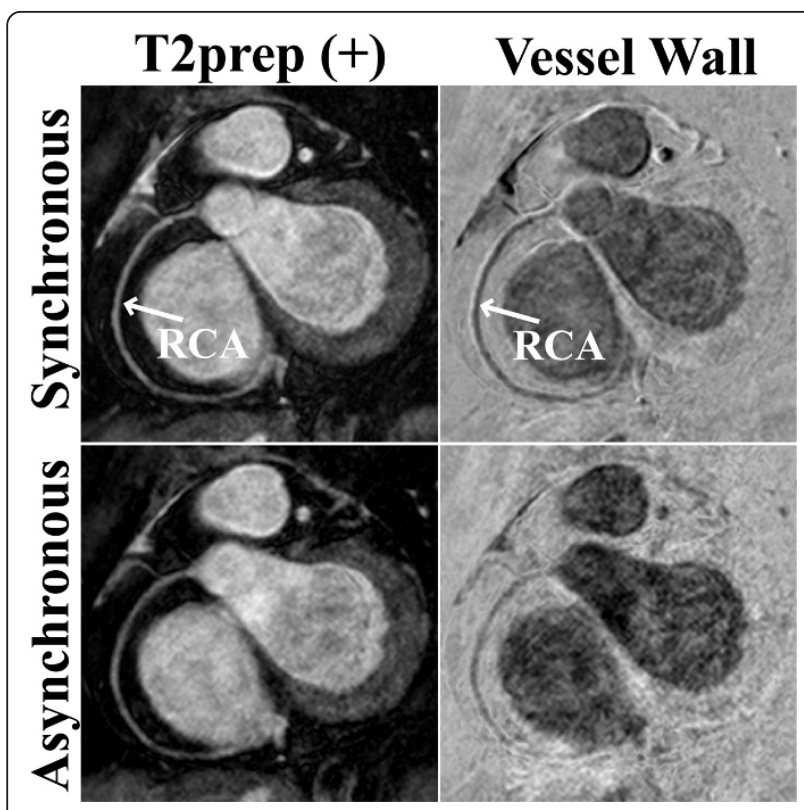

Figure 2 Reformatted images from targeted RCA acquisitions using an interleaved T2prep (i-T2prep) sequence with synchronous and asynchronous navigating techniques, depicting the interleaf with T2prep (T2prep(+)), and the coronary vessel wall image acquired via weighted subtraction of the two interleaves. While the T2prep(+) images are of similar quality, the synchronous technique yields a better depiction of the coronary vessel wall. The asynchronous technique is affected by motion within the $5 \mathrm{~mm}$ gating window between the two interleaves. The synchronous scheme results in a longer acquisition (20:31 vs. 8:15 minutes).

asynchronous scheme is shorter (8:03 vs. 11:58 mins). Figure 2 shows reformatted RCA images from another volunteer. The synchronous scheme has superior image quality, but also a longer acquisition time (20:31 vs. 8:15 mins). The synchronous scheme provides significantly better delineated vessel walls in terms of vessel sharpness $(0.15 \pm 0.02$ vs. $0.13 \pm 0.02, \mathrm{P}=0.03)$ although the absolute difference is small. However, the asynchronous scheme is significantly shorter (9:29 $\pm 1: 35$ vs. 18:18 $\pm 6: 21$ mins, $\mathrm{P}=0.005)$.

\section{Conclusions}

We have investigated two navigating approaches for high-resolution interleaved sequences. Examples and quantitative data show that while the asynchronous scheme allows a significantly shorter scan time, independently accepting data from interleaves results in loss of image quality and sharpness, even though a $5 \mathrm{~mm}$ gating window is utilized. Techniques that require voxelby-voxel processing of images from multiple interleaves may require motion correction within the gating window if a synchronous scheme cannot be utilized due to a higher number of interleaves.

\section{Funding}

NIH:K99HL111410-01;R01EB008743-01A2; BHF: RG/ $12 / 1 / 29262$.

\section{Author details}

${ }^{1}$ Medicine, Beth Israel Deaconess Medical Center, Harvard Medical School, Boston, MA, USA. ${ }^{2}$ Imaging Sciences \& Biomedical Engineering, King's College London, London, UK.

Published: 30 January 2013

\author{
References \\ 1. Kellman:: MRM 2003. \\ 2. Andia: MRM 2012 \\ 3. Mehta:. JCMR 2012.
}

doi:10.1186/1532-429X-15-S1-E20

Cite this article as: Akcakaya et al.: Comparison of respiratory navigator techniques for interleaved high-resolution coronary vessel wall imaging. Journal of Cardiovascular Magnetic Resonance 2013 15(Suppl 1):E20.

\section{Submit your next manuscript to BioMed Central and take full advantage of:}

- Convenient online submission

- Thorough peer review

- No space constraints or color figure charges

- Immediate publication on acceptance

- Inclusion in PubMed, CAS, Scopus and Google Scholar

- Research which is freely available for redistribution
Biomed Central 